\title{
Enantioselective Diels-Alder Reactions of Carboxylic Ester Dienophiles Catalysed by Titanium-Based Chiral Lewis Acid
}

\author{
YOGESH K. CHOUGHULE and ANAND V. PATWARDHAN* \\ Department of Chemical Engineering, Institute of Chemical Technology, \\ Matunga, Mumbai 400019, Maharashtra, India. \\ *Corresponding author E-mail: av.patwardhan @ ictmumbai.edu.in
}

http://dx.doi.org/10.13005/ojc/320217

(Received: March 12, 2016; Accepted: April 05, 2016)

\begin{abstract}
A new titanium-based chiral Lewis acid 1 has been developed using (1R,2R)-1,2-bis-(2methoxyphenyl)-ethane-1,2-diol as a chiral vicinal diol ligand. This chiral catalyst was found to exhibit uniformly high enantioselectivity towards carboxylic ester dienophiles in Diels-Alder reactions. The chiral vicinal ligand (1R,2R)-1,2-bis-(2-methoxyphenyl)-ethane-1,2-diol is inexpensive and is easily accessible.
\end{abstract}

Keyword: Chiral Lewis acid, Chiral vicinal ligand, Diels-Alder reaction, Dienophiles, Carboxylic esters.

\section{INTRODUCTION}

The Diels-Alder reaction is known to be a popular method in synthetic chemistry. ${ }^{1}$ The control of absolute stereochemistry has generated a great interest recently. ${ }^{2}$ One successful methodology developed thus far utilises chiral auxiliaries that are integrated into the substrate, and eventually detached from the product by some chemical means. Here, the stereochemical consequence is due to the absolute stereocentre of the substrate (substrate control). There is an alternative method, which has not yet been as exhaustively explored, and which involves introduction of the stereocentre on the reagent (reagent control). ${ }^{3,4}$ This involves the cycloaddition of dienes and dienophiles catalysed by chiral Lewis acids, and simultaneous induction of asymmetry into the product. An example of such type of work, which involve highly reactive oxazolidinone dienophiles were successfully achieved by Narasaka, Chapuis, and Corey ${ }^{4,5}$ However, the carboxylic ester dienophiles have shown poor enantioselectivity under these reagent-controlled conditions because of their less reactivity. The present work describes an attempt to develop a chiral Lewis acid for DielsAlder reactions which induces asymmetry with ester dienophiles. 


\section{MATERIALS AND METHODS}

\section{General information}

Dichloromethane was purified by calcium hydride distillation. Diethyl ether was distilled from potassium hydroxide and purified by sodium wire. TLC and column chromatography were carried out with silica gel. All reactions were conducted under nitrogen atmosphere, and the product was concentrated under reduced pressure with a rotary evaporator. The purity and characterisation of all compounds were done using HPLC and NMR techniques.

\section{General procedure for titanium-based chiral} Lewis acid formation 1

A $100 \mathrm{~mL}$ 3-neck round bottom flask was fitted with calcium guard tube, nitrogen gas inlet, and rubber septum respectively, to maintain anhydrous conditions. n-butyllithium $(1.68 \mathrm{~mL}$ of $2.5 \mathrm{M}$ in hexane, $4.2 \mathrm{mmol}$ ) was added to a suspension of (1R,2R)-1,2-bis-(2-methoxyphenyl)-ethane-1,2-diol $(0.575 \mathrm{~g}, 2.1 \mathrm{mmol})$ in $4 \mathrm{~mL}$ of diethyl ether at $0^{\circ} \mathrm{C}$. The resulting slurry was dissolved in $16 \mathrm{~mL}$ of solvent (different solvents were used for effect of solvent experiments see Table 3), and titanium tetrachloride (2.1 $\mathrm{mL}$ of $1.0 \mathrm{M}$ solution in Dichloromethane, 2.1 $\mathrm{mmol}$ ) was added. Lithium Chloride got precipitated. This mixture was used as such.

\section{General procedure for Diels-Alder reaction}

Dienophile $(1.4 \mathrm{mmol})$ was added to a mixture of a Lewis acid (as prepared above), followed by diene $(7 \mathrm{mmol})$. The resulting mixture was stirred at $25{ }^{\circ} \mathrm{C}$ (carried out at different temperatures for temperature study: Table 2) for 4 $\mathrm{h}$, and was quenched with $10 \mathrm{~mL}$ of $1 \mathrm{~N}$ aqueous hydrochloric acid. The aqueous phase was extracted ( $3 \times 20 \mathrm{~mL}$ of dichloromethane). The combined organic phase were dried (using anhydrous sodium sulphate) and concentrated on rotary evaporator. Column chromatography of crude oil (silica, 9.5:0.5 hexane:ethyl acetate) gives enantioenriched DielsAlder product as colourless oil.

Dimethyl-(4S,5S)-1,2-dimethylcyclohexene-4,5dicarboxylate (2a)

${ }^{1} \mathrm{H} \mathrm{NMR}\left(300 \mathrm{MHz}, \mathrm{CDCl}_{3} ; \delta, \mathrm{ppm}\right) 1.56$ (s, $6 \mathrm{H}), 2.05-2.23(\mathrm{~m}, 4 \mathrm{H}), 2.74-2.78(\mathrm{~m}, 2 \mathrm{H}), 3.63(\mathrm{~s}$, $6 \mathrm{H}) .{ }^{13} \mathrm{C} \mathrm{NMR}\left(75 \mathrm{MHz}, \mathrm{CDCl}_{3} ; \delta, \mathrm{ppm}\right) 175.2,123.7$, 51.6, 41.8, 34.0, 18.4.

\section{Dimethyl-(4S,5S)-I-methylcyclohexene-4,5-} dicarboxylate (2b)

${ }^{1} \mathrm{H}$ NMR $\left(300 \mathrm{MHz}, \mathrm{CDCl}_{3} ; \delta, \mathrm{ppm}\right) 1.64$ (s, 3H), 2.06-2.38 (m, 4H), $2.75(\mathrm{dt}, J=5.3,10.8$ $\mathrm{Hz}, 1 \mathrm{H}), 2.85(\mathrm{dt}, J=5.2,10.8 \mathrm{~Hz}, 1 \mathrm{H}), 3.65(\mathrm{~s}, 3 \mathrm{H})$, $3.66(\mathrm{~s}, 3 \mathrm{H}), 5.33(\mathrm{~s}, 1 \mathrm{H}) \cdot{ }^{13} \mathrm{C} \mathrm{NMR}\left(75 \mathrm{MHz}, \mathrm{CDCl}_{3}\right.$; $\delta$, ppm) $175.3,175.2,132.1,118.9,52.0,51.7,41.6$, 41.0, 32.4, 27.96, 22.88.

\section{Dimethyl-(4S,5S)-cyclohexene-4,5-dicarboxylate} (2c)

${ }^{1} \mathrm{H}$ NMR $\left(300 \mathrm{MHz}, \mathrm{CDCl}_{3} ; \delta, \mathrm{ppm}\right)$ 2.12$2.19(\mathrm{~m}, 2 \mathrm{H}), 2.36-2.43(\mathrm{~m}, 2 \mathrm{H}), 2.81-2.85(\mathrm{~m}$, $2 \mathrm{H}), 3.67(\mathrm{~s}, 6 \mathrm{H}), 5.66(\mathrm{~d}, \mathrm{~J}=2.3 \mathrm{~Hz}, 2 \mathrm{H}) \cdot{ }^{13} \mathrm{C}$ NMR $\left(75 \mathrm{MHz}, \mathrm{CDCl}_{3} ; \delta, \mathrm{ppm}\right) 175.2,124.8,51.8,41.1$, 27.8.

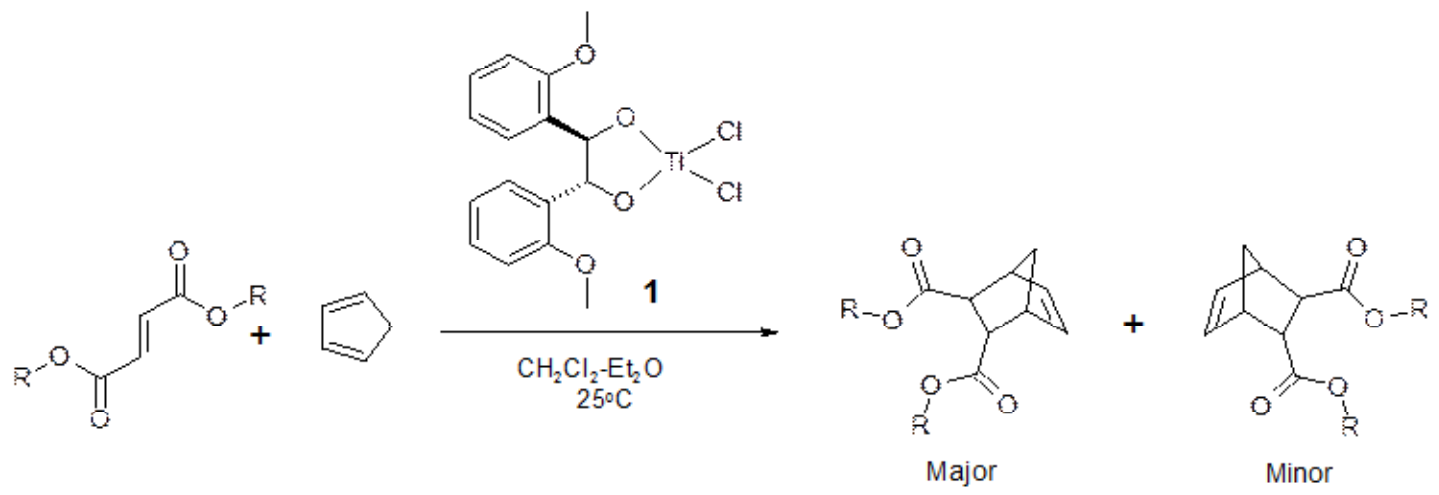

Fig. 1: Diels-Alder reactions catalysed by Lewis acid 1 
Table 1: Enantioselective Diels-Alder reactions catalysed by the titanium-based chiral Lewis acid $1\left(\mathrm{~T}=25^{\circ} \mathrm{C}, 4 \mathrm{~h}\right)$

\begin{tabular}{|c|c|c|c|c|c|}
\hline Entry & Diene & Dienophile & Major Product & $e^{a \%}$ & Isolated Yield ${ }^{\mathrm{b}} \%$ \\
\hline 1 & & & & 94 & 82 \\
\hline 2 & & & & 92 & 80 \\
\hline 3 & & & & $76-80^{c}$ & $55-78^{c}$ \\
\hline 4 & & & & 90 & 79 \\
\hline 5 & & & & 77 & 84 \\
\hline 6 & & & & 71 & 82 \\
\hline 7 & & & & $60-64^{c}$ & $58-80^{c}$ \\
\hline 8 & & & & 68 & 81 \\
\hline 9 & & & & 60 & 89 \\
\hline 10 & & & & 55 & 87 \\
\hline 11 & & & & $45-48^{c}$ & $60-84^{c}$ \\
\hline 12 & & & & 50 & 85 \\
\hline
\end{tabular}

${ }^{a}$ Enantiomeric excess (ee\%) was determined by chiral HPLC column CHIRALPAK IA analytical 250×4.6 mm, $5 \mu \mathrm{m}$ (at $254 \mathrm{~nm}$, methanol)

${ }^{b}$ Isolated yield by column chromatography (silica gel, $5 \%$ ethyl acetate in hexane)

'The reaction was conducted at $-10^{\circ} \mathrm{C}$. 
Dimethyl-(2S,3S)-bicyclo[2.2.I]hept-5-ene-2,3dicarboxylate (2d)

${ }^{1} \mathrm{H}$ NMR $\left(300 \mathrm{MHz}, \mathrm{CDCl}_{3} ; \delta, \mathrm{ppm}\right) 1.46$ (dd, $J=1.5 \mathrm{~Hz}, 8.9 \mathrm{~Hz}, 1 \mathrm{H}), 1.62(\mathrm{~d}, J=8.9 \mathrm{~Hz} 1 \mathrm{H})$, 2.69 (dd, $J=1.3,4.1 \mathrm{~Hz}, 1 \mathrm{H}), 3.13(\mathrm{br} \mathrm{s}, 1 \mathrm{H}), 3.27$ (br s, 1H), 3.38 (t, J=4.0 Hz, 1H), 3.65 (s, 3H), 3.72 (s, 3H), 6.07 (dd, J=2.7, $5.5 \mathrm{~Hz}, 1 \mathrm{H}$ ), 6.29 (dd, $J=$ 3.2, $5.3 \mathrm{~Hz}, 1 \mathrm{H},) .{ }^{13} \mathrm{C} \mathrm{NMR}\left(75 \mathrm{MHz}, \mathrm{CDCl}_{3} ; \delta, \mathrm{ppm}\right)$ 174.7, 173.5, 137.4, 135.0, 52.0, 51.7, 47.7, 47.5, $47.2,46.9,45.5$.

\section{Methyl-(1S,6S)-3,4,6-trimethylcyclohex-3-ene-1- carboxylate $(2 \mathrm{e})$}

${ }^{1} \mathrm{H}$ NMR $\left(300 \mathrm{MHz}, \mathrm{CDCl}_{3} ; \delta\right.$, ppm) 0.81 (d, 3H), $1.22(\mathrm{~m}, 1 \mathrm{H}), 1.63(\mathrm{~s}, 3 \mathrm{H}), 1.74(\mathrm{~s}, 3 \mathrm{H}), 2.07-$ $2.40(\mathrm{~m}, 4 \mathrm{H}), 2.80-2.84(\mathrm{~m}, 1 \mathrm{H}), 3.70(\mathrm{~s}, 3 \mathrm{H}) .{ }^{13} \mathrm{C}$ NMR $\left(75 \mathrm{MHz}, \mathrm{CDCl}_{3} ; \delta, \mathrm{ppm}\right) 175.3,132.4,129.7$, 51.8, 41.6, 33.0, 32.4, 27.9, 23.1, 22.9, 20.1.

\section{Methyl-(1S,6S)-4,6-dimethylcyclohex-3-ene-1- carboxylate (2f)}

${ }^{1} \mathrm{H}$ NMR $\left(300 \mathrm{MHz}, \mathrm{CDCl}_{3} ; \delta, \mathrm{ppm}\right) 0.91$ (d, $3 \mathrm{H}), 1.25(\mathrm{~m}, 1 \mathrm{H}), 1.66(\mathrm{~s}, 3 \mathrm{H}), 2.10-2.43(\mathrm{~m}, 4 \mathrm{H})$, 2.82-2.87 (m, 1H), $3.73(\mathrm{~s}, 3 \mathrm{H}), 5.35(\mathrm{t}, 1 \mathrm{H}) .{ }^{13} \mathrm{C}$ NMR $\left(75 \mathrm{MHz}, \mathrm{CDCl}_{3} ; \delta, \mathrm{ppm}\right) 179.3,130.7,118.0,52.2$, 45.6, 37.0, 36.1, 31.9, 25.1, 22.1.

\section{Methyl-(1S,6S)-6-methylcyclohex-3-ene-1-} carbooxylate $(2 \mathrm{~g})$

${ }^{1} \mathrm{H}$ NMR (300 MHz, $\left.\mathrm{CDCl}_{3} ; \delta, \mathrm{ppm}\right) 0.88(\mathrm{~d}$, $3 \mathrm{H}), 1.23(\mathrm{~m}, 1 \mathrm{H}), 2.11-2.44(\mathrm{~m}, 4 \mathrm{H}), 2.80-2.85(\mathrm{~m}$, $1 \mathrm{H}), 3.71(\mathrm{~s}, 3 \mathrm{H}), 5.33(\mathrm{~m}, 1 \mathrm{H}), 5.63(\mathrm{~m}, 1 \mathrm{H}) .{ }^{13} \mathrm{C}$ NMR (75 MHz, $\left.\mathrm{CDCl}_{3} ; \delta, \mathrm{ppm}\right) 179.3,120.7,118.0$, 52.2, 45.6, 37.0, 36.1, 31.9, 22.1.

Methyl-(2S)-3-methyl(3S)bicyclo[2.2.1] hept-5ene-2-carboxylate (2h)

${ }^{1} \mathrm{H}$ NMR (300 MHz, $\left.\mathrm{CDCl}_{3} ; \delta, \mathrm{ppm}\right) 1.17$ (d, $3 \mathrm{H}), 1.78(\mathrm{~m}, 3 \mathrm{H}), 2.27(\mathrm{~m}, 2 \mathrm{H}), 2.42(\mathrm{~m}, 1 \mathrm{H}), 3.05$ $(\mathrm{m}, 1 \mathrm{H}), 3.27(\mathrm{~m}, 1 \mathrm{H}), 3.57(\mathrm{~s}, 3 \mathrm{H}), 5.91(\mathrm{dd}, 1 \mathrm{H})$, $6.18(\mathrm{dd}, 1 \mathrm{H}) .{ }^{13} \mathrm{C}$ NMR $\left(75 \mathrm{MHz}, \mathrm{CDCl}_{3} ; \delta, \mathrm{ppm}\right)$ 175.2, 138.4, 134.6, 51.5, 47.8, 47.1, 46.8, 45.4, $29.2,21.1$.

Methyl-(1R)-3,4-dimethylcyclohex-3-ene-1carboxylate (2i)

${ }^{1} \mathrm{H}$ NMR (300 MHz, $\left.\mathrm{CDCl}_{3} ; \delta, \mathrm{ppm}\right) 1.60$ (s, $6 \mathrm{H}), 1.61-1.69(\mathrm{~m}, 1 \mathrm{H}), 1.90-2.25(\mathrm{~m}, 5 \mathrm{H}), 2.49-$ $2.58(\mathrm{~m}, 1 \mathrm{H}), 3.67(\mathrm{~s}, 3 \mathrm{H}) .{ }^{13} \mathrm{C} \mathrm{NMR}\left(75 \mathrm{MHz}, \mathrm{CDCl}_{3}\right.$; $\delta$, ppm) 176.6, 125.4, 124.0, 51.7, 40.2, 33.8, 31.1, 26.0, 19.1, 18.9.

\section{Methyl-(4R)-1-methylcyclohexene-4-carboxylate} (2j)

${ }^{1} \mathrm{H}$ NMR (300 MHz, $\left.\mathrm{CDCl}_{3} ; \delta, \mathrm{ppm}\right)$ 1.59$1.62(\mathrm{~m}, 2 \mathrm{H}), 1.65(\mathrm{~s}, 3 \mathrm{H}), 1.98-2.02(\mathrm{~m}, 2 \mathrm{H}), 2.21$ $2.23(\mathrm{~m}, 2 \mathrm{H}), 2.45-2.53(\mathrm{~m}, 1 \mathrm{H}), 3.68(\mathrm{~s}, 3 \mathrm{H}), 5.38$ (s, $1 \mathrm{H}) .{ }^{13} \mathrm{C}$ NMR $\left(75 \mathrm{MHz}, \mathrm{CDCl}_{3} ; \delta, \mathrm{ppm}\right) 176.5$, 133.7 119.2, 51.6, 39.1, 29.3, 27.7, 25.5, 23.5.

Methyl-(1R)-cyclohex-3-ene-1-carboxylate (2k)

${ }^{1} \mathrm{H}$ NMR (300 MHz, $\left.\mathrm{CDCl}_{3} ; \delta, \mathrm{ppm}\right) 1.73-$ $1.65(\mathrm{~m}, 2 \mathrm{H}, \mathrm{CH} 2), 2.15-1.99(\mathrm{~m}, 2 \mathrm{H}, \mathrm{CH} 2), 2.26-$ 2.25 (m, 2H, CH2), 2.60-2.55 (m, 1H, CHCO2Me), $3.70(\mathrm{~s}, 3 \mathrm{H}, \mathrm{CO} 2 \mathrm{CH} 3), 5.72-5.65(\mathrm{~m}, 2 \mathrm{H}, \mathrm{CH}=\mathrm{CH})$, ${ }^{13} \mathrm{C}$ NMR $\left(75 \mathrm{MHz}, \mathrm{CDCl}_{3} ; \delta, \mathrm{ppm}\right) 176.6,126.9$, 125.4, 51.9, 39.4, 27.7, 25.3, 24.7.

\section{Methyl bicyclo[2.2.1]hept-5-ene-2-carboxylate} (2I)

${ }^{1} \mathrm{H} \mathrm{NMR}\left(300 \mathrm{MHz}, \mathrm{CDCl}_{3} ; \delta, \mathrm{ppm}\right) 1.26(\mathrm{~m}$, $1 \mathrm{H}), 1.41(\mathrm{~m}, 1 \mathrm{H}), 1.43(\mathrm{~m}, 1 \mathrm{H}), 1.90(\mathrm{~m}, 1 \mathrm{H}), 2.89$ $(\mathrm{m}, 1 \mathrm{H}), 2.93(\mathrm{~m}, 1 \mathrm{H}), 3.19(\mathrm{~m}, 1 \mathrm{H}), 3.64(\mathrm{~s}, 3 \mathrm{H}), 5.95$ (dd, $1 \mathrm{H}), 6.16$ (dd, $1 \mathrm{H}) .{ }^{13} \mathrm{C} \mathrm{NMR}\left(75 \mathrm{MHz}, \mathrm{CDCl}_{3}\right.$; $\delta$, ppm) 172.2, 134.0, 128.7, 53.4, 49.0, 42.2, 39.0, 38.8, 26.3.

Table 2: Enantioselective Diels-Alder reactions catalysed by the titanium-based chiral Lewis acid 1 carried out at different temperature ${ }^{a}$

\begin{tabular}{lccc}
\hline Entry & Temp. $^{\mathrm{d}}\left({ }^{\circ} \mathbf{C}\right)$ & ee $^{\mathrm{b} \%}$ & Yield $^{\mathrm{c} \%}$ \\
1 & -10 & 94 & 70 \\
2 & 0 & 94 & 79 \\
3 & 10 & 94 & 80 \\
4 & 25 & 94 & 82 \\
5 & 30 & 80 & 84 \\
\hline
\end{tabular}

${ }^{a}$ All experiments were carried out using diene and dienophile mentioned in (Table 1, entry 1).

${ }^{b}$ Enantiomeric excess (ee\%) was determined by chiral HPLC column Chiralpak IA analytical $250 \times 4.6 \mathrm{~mm}, 5 \mu \mathrm{m}$ (at $254 \mathrm{~nm}$, methanol)

'Yield of racemic mixtures were determined by HPLC

${ }^{d}$ Entries no.1, 2, 3, experiments were carried out for 12, 8, $6 \mathrm{~h}$ respectively and entries 4,5 for $4 \mathrm{~h}$. 
Table 3: Enantioselective Diels-Alder reactions catalysed by the titanium-based chiral Lewis acid 1 carried out using different solvents $^{\mathrm{a}}$

\begin{tabular}{lccc}
\hline Entry & Solvent & ee $^{\mathrm{b}} \%$ & Yield $^{\mathrm{c}} \%$ \\
\hline 1 & Dichloromethane & 94 & 82 \\
2 & 1,2-Dichloroethane & 90 & 80 \\
3 & Benzene & 60 & 84 \\
4 & Toluene & 66 & 80 \\
5 & Xylene & 68 & 78 \\
6 & n-Hexane & 26 & 88 \\
\hline
\end{tabular}

${ }^{\text {aAll }}$ experiments were carried out at $25^{\circ} \mathrm{C}$ for $4 \mathrm{~h}$ using diene and dienophile mentioned in (Table 1, entry 1). ${ }^{b}$ Enantiomeric excess (ee\%) was determined by chiral HPLC column CHIRALPAK IA analytical $250 \times 4.6 \mathrm{~mm}$, $5 \mu \mathrm{m}$ (at $254 \mathrm{~nm}$, methanol)

'Yield of racemic mixtures were determined by HPLC.

\section{RESULTS AND DISCUSSION}

A review of several $\mathrm{C}_{2}$-symmetric diol ligands employing boron, tin, and titanium Lewis acids encouraged us to select a titanium-based Lewis acid of type 1 (Fig. 1), in which optically active (1R,2R)-1,2-bis-(2-methoxyphenyl)-ethane-1,2-diol was used as the ligand. ${ }^{6}$ this optically active ligand was easily synthesised using Sharpless osmylation method. ${ }^{7}$ The outcome of the Diels-Alder reactions as illustrated by (Fig. 1) is compiled in (Table 1).

The chiral Lewis acid 1 was generated by transformation of (1R,2R)-1,2-bis-(2-methoxy phenyl)-ethane-1,2-diol to the corresponding dilithiodialkoxide species upon reaction with two equivalents of n-butyllithium in diethyl ether. The resulted mixture was then diluted with dichloromethane, and titanium tetrachloride was added. At this point, lithium chloride got precipitated. ${ }^{8}$ Then the dienophile and diene were added respectively. The reactions were normally carried out for $4 \mathrm{~h}$ and monitored by TLC and purified by silica gel column chromatography using 200-400 mesh particle size of S D Fine-Chem Ltd., Mumbai. The (1R,2R)-1,2-bis-(2-methoxyphenyl)-ethane-1,2-diol was readily recovered in near quantitative amounts. A slight decomposition was observed leading to traces of 2-methoxy-benzaldehyde. The enantioselectivity of products was analyzed by HPLC technique using a chiral column. The results were certainly promising for high enantioselectivity towards Diels-Alder reaction by a titanium-based chiral Lewis acid as a chiral catalyst (compound-1 in Fig. 1). Results for the Diels-Alder reaction are summarised in (Table 1). The configuration of the resulted product (2a-2I) was confirmed by reported literature. ${ }^{9}$ In all these cases the product was conveniently isolated by column chromatography. It is interesting to note that $(1 \mathrm{R}, 2 \mathrm{R})$ 1,2-bis-(2-methoxyphenyl)-ethane-1,2-diol examined as ligands and gave reasonable enantiomeric yield. The results of temperature and solvent experiments summarized in (table 2) and (table 3) respectively has been showed optimum enatiomeric and racemic yield at $25{ }^{\circ} \mathrm{C}$ when dichloromethane solvent was used.

\section{CONCLUSIONS}

In this work, it was found that high enantioselectivities are possible for Diels-Alder reaction of carboxylic esters catalysed by titaniumbased chiral Lewis acid. It may be happening because of availability of the lone pair of electrons on the oxygen atoms present in the carboxylic ester group. The lone pair of electrons may be leading to more steric hindrance in the transition state of reaction. This resulted in high enantioselectivity of the product. (1R,2R)-1,2-bis-(2-methoxyphenyl)ethane-1,2-diol, a simple and readily available compound, was effective as the chiral ligand when titanium metal was used in the Lewis acid. The titanium is a transition metal element located in the fourth group of fourth period in the periodic table. Because of typical electronic structure of transition elements, they have the ability to adopt multiple oxidation states to form complexes. They are more effective catalysts because they have the ability to make complexes with non-bonding electron donating groups like carboxylic esters group in the dienophile (see Dienophile in Table 1). Titanium has [Ar]3d24s2 electronic configuration having valence d-orbital which might be responsible to make more sterically hindered complexes in the transition state of reaction. The temperature (Table 2) and solvent (Table 3) studies showed that $25^{\circ} \mathrm{C}$ was an optimum temperature and dichloromethane was found to be the best solvent for this type of Diels-Alder reaction when titanium-based chiral Lewis acid $\mathbf{1}$ was used. 


\section{ACKNOWLEDGEMENTS}

The author (YKC) is thankful to University Grants Commission (UGC) - New Delhi, India, for the award of 'senior research fellowship'. We are also thankful to Dr. Sandeep B. Kotwal, Assistant Professor, Department of Chemistry, CHM College, University of Mumbai, for his willing help.

\section{REFERENCES}

1. (a) Desmoni, G.; Tacconi, G.:, Pollini, G.; Natural Product Synthesis Through Pericyclic Reactions, ACS Monograph 180, American Chemical Society, Washington, DC, 1984, Chapter 5. (b) Johari, N. L. S.; Hassan, N. H., Hassan, N. I., Orient. J. Chem, 2014, 30, 1191-1196.(c) Al-Saeedi, A., Farooqui, M.; Orient. J. Chem, 2013, 29, 1033-1039.(d) Alamdari, R. F., Zamani, F. G., Shekarriz, M.; Orient. J. Chem, 2015, 31, 1127-1132.

2. (a) Taeboem, O.; J. Org. Chem. 1992, 57, 396-399. (b) Ryu, D. H.; Corey, E. J.; J. Am. Chem. Soc. 2003, 125, 6388-6390. (c) Bayer, A.; Hansen, L. K.; Gautun, O. R.; Tetrahedron: Asymmetry, 2002, 13, 24072415. (d) Manickam, G.; Sundararajan, G.; Tetrahedron: Asymmetry, 1999, 10, 29132925. (e) Evans, D. A.; Barnes, D. M.; J. Am. Chem. Soc. 2009, 121, 7582-7594. (f) Bhowmick, K. C.; Joshi, N. N.; Tetrahedron: Asymmetry, 2006, 17, 1901-1929. (g) Singh, R. S.; Harada, T.; J. Org. Chem. 2008, 73, 212-218. (h) Sakakura, A.; Ishihara, K.; J. Am. Chem. Soc. 2009, 131, 17762-17764. (i) Evans, D. A.; Chapman, K. T.; Bisaha, J.; J. Am. Chem. Soc. 1988, 110, 1238-1256.

3. (a) Narasaka, K.; Synthesis, 1991, 1. (b) Tomioka, K.; Synthesis, 1990, 542.

4. (a) Rebiere, F.; Kagan, G. B.; Tetrahedron Asymmetry, 1990, 1, 199-214. (b) Narasaka, K.; Iwasawa, N.; Inoue, M.; Yamada, T.; Nakashima M.; Sugimori, J.; J. Am. Chem. Soc. 1989, 111, 5340-5345. (c) Chapuis, C.; Jurczak, J.; Helv. Chim. Acta., 1987, 70, 436440. (d) Corey, E. J.; Imwinkelried, R.; Pikul,
S.; Xiang, Y. B.; J. Am. Chem. Soc. 1989, 111, 5493-5495. (e) Y. Hayashi, K. Narasaka, Chem. Lett. 1989, 18, 793-796. (f) Bednarski, M.; Maring, B.; Danishefsky, S.; Tetrahedron Lett. 1983, 24, 3451-3454. (g) Marvoka, K.; Sakurai, M.; Fujiwara, J.; Yamamoto, H.; Tetrahedron Lett. 1986, 27, 4895-4898. (h) Corey, E. J.; Imai, N.; Zhang, H. Y.; J. Am. Chem. Soc. 1991, 113, 728-729.

5. Evans, D. A.; Chapman, K. T.; Bisaha, J.; J. Am. Chem. Soc. 1988, 110, 1238-1256.

6. (a) Whitesell, J. K.; Chem. Rev. 1989, 89, 1581-1590. (b) Alexakis, A.; Mangeney, P.; Tetrahedron Asymmetry, 1990, 1, 477-511.

7. Jacobsen, E. N.; Marko, I.; Mungall, W. S.; Schroder, G.; Sharpless K. B.; J. Am. Chem. Soc. 1988, 110, 1968-1970.

8. (a) Seebach, D.; Weidmann, B.; Widler, L.; Modern Synthetic Methods, Scheffold, R., Ed., John Wiley and Sons, New York, 1983, 3, 217. (b) Seebach, D.; Beck, A. K.; Imwinkelried, R.; Roggo, S.; Wonnacott, A.; Helv. Chim. Acta. 1987, 70, 954-974. (c) Reetz, M. T.; Kyung, S. H.; Bolm, C.; Zierke, T.; Chem. Ind. 1986, 824-826.

9. (a) Corey, E. J.; J. Am. Chem. Soc. 2007, 129, 1498-1499. (b) Silvia, M.; Eur. J. Org. Chem. 2004, 24, 5119-5225. (c) Virgili, A.; J. Org. Chem. 2006, 71, 3267-3269. (d) Monnin; Helv. Chim. Acta. 1958, 41, 2112-2119. (e) Jerome, A.; J. Am. Chem. Soc. 1961, 83, 4947-5956. (f) Oestreich, M.; Angew. Chem. Int. Ed. 2009, 48, 9077-9079. (g) Tanyeli, C; Tetrahedron: Asymmetry, 2004, 15, 2057-2060. (h) Berson, J. A.; J. Am. Chem. Soc. 1959, 81, 40834087. 